\title{
The distribution of hand fractures at the University Hospital of North Norway
}

\section{Running title}

Hand fractures in Northern Norway

1,2Sven Weum, M.D. Ph.D.

1,2Siri Millerjord, M.D.

1,3Louis de Weerd, M.D. Ph.D.

${ }^{1}$ Medical Imaging Research Group

Department of Clinical Medicine

UiT The Arctic University of Norway

9037 Tromsø, Norway

${ }^{2}$ Department of Radiology

University Hospital of North Norway

P.O. Box 103

9038 Tromsø, Norway

${ }^{3}$ Department of Plastic Surgery and Hand Surgery

University Hospital of North Norway

9038 Tromsø, Norway 
Hand fractures in Northern Norway

\section{Corresponding author}

Sven Weum, M.D. Ph.D.

Associate Professor

Department of Radiology

University Hospital of North Norway

P.O. Box 103

9038 Tromsø, Norway

Phone: +47 77628311

E-mail: sven.weum@unn.no

(sven.weum@unn.no) 


\section{ABSTRACT}

\section{Background}

The objective of this study was to describe the fracture pattern for hand fractures in the Arctic region of Norway.

\section{Material and methods}

Using the hospital's radiological information system and picture archiving and communication system all traumatic hand fractures in the year of 2010 were registered. Fractures in the carpal, metacarpal and phalangeal bones were included in the study.

\section{Results}

A total of 593 patients with 691 fractures including 80 carpal, 200 metacarpal and 411 phalangeal fractures were registered. The most commonly affected bone was the $5^{\text {th }}$ metacarpal bone with a total of 99 fractures $(14 \%), 22$ of these $(22$ \%) were classified as subcapital or "boxer's fracture". Among male patients, the highest frequency of fractures was seen in the age group 10 to 60 years with a peak between 10 and 24 years. Adult female patients had most fractures in the age group 50 to 70 years. Generally, there were no seasonal variations, although a marked decrease of fractures could be seen in December. Triquetral fractures were more common during the winter season. Falling was the most common trauma mechanism. 
Hand fractures in Northern Norway

\section{Conclusion}

With a few exceptions, hand fractures were not related to particular seasons or weekdays in this material. The distribution of hand fractures was related to age and gender and some trauma mechanisms were dominating.

\section{Keywords}

Hand; Trauma 


\section{Introduction}

Studies on the distribution of hand fractures have been performed in Bergen [1] and Amsterdam [2] but not in Northern Norway. Several factors may influence the prevalence and distribution of hand fractures.

Certain trauma mechanisms are dominating causes of hand fractures. Falling has been shown to be a common cause of hand fractures in both genders and in all age groups, especially among children and women [3-5], while fractures caused by violence and aggression are more common among men [4, 6]. Mahabir et al. reported that $58 \%$ of all hand fractures in children were caused by sport [7], while a study by Feehan et al. reported that ball games are causing $35 \%$ of all hand fractures in children [8].

Previous studies have shown higher incidence of hand fractures among males than females between the age of 10 and 60 years $[2,4,5,8]$. Hand fractures are most common among people between 10 and 40 years of age $[2,4,8]$. Occupational injuries are reported to cause approximately $9 \%$ of the hand fractures $[3,9]$. Men in the age group 16 to 45 years are most often affected by occupational hand fractures. Fingertips are more often injured than the rest of the hand [10], often in association to the use of tools and machines $[10,11]$.

There are also studies that have reported on seasonal variations in the distribution of hand fractures [2]. The University Hospital of North Norway serves a population of 480.740 in the Arctic region of Norway. Long winters, 
polar darkness and slippery roads due to snow and ice characterize this area. Knowledge on the causes of hand fractures may contribute to take preventive measures. The objective of this study was to describe the fracture pattern for hand fractures in the Arctic region of Norway.

\section{Materials and methods}

All new hand fractures in the radiological information system (RIS) and picture archiving and communication system (PACS) between January the $1^{\text {st }}$ and December the $31^{\text {st }} 2010$ were registered. Control examinations of traumas occurring before January the $1^{\text {st }} 2010$ were excluded from the study. Fractures in the carpal, metacarpal and phalangeal bones were included. Cases where fracture could neither be ruled out nor confirmed were excluded. All images from examinations registered as fractures in the RIS were reread and the fractures classified according to anatomical localization. All images had primarily been read and described by senior radiologists. A fifth year medical student reread the referral texts and categorized the fractures based on the original radiological images and descriptions as a part of a student research project. In case of doubt, the student consulted the supervisor, a senior radiologist. In addition to specifying right or left hand, the involved bones were registered. Based on rereading of the radiographic images and their descriptions, metacarpal and phalangeal fractures were classified as basal, diaphysal, subcapital, capital, intra-articular, extra-articular or epiphyseal fractures. Date of injury, patient age, gender, trauma mechanism (including aggression, confirmed sport- or occupational injury), anatomical localization and information regarding 
fracture reduction or surgical intervention were registered in all cases. In cases where the exact date of injury was missing, an estimate was based on information in the referral text. In a number of cases patients were transferred from other hospitals with no information on trauma mechanism. In these cases no trauma mechanism was registered. All data were depersonalized and registered in a Microsoft Excel spreadsheet (Excel for Mac 2011, Microsoft Inc., Seattle, WA, USA). Descriptive statistics and analysis of frequencies and distribution of fractures in relation to other variables were performed using SPSS (version 22, IBM Corp., Somers, NY, USA) to analyze frequencies and distribution of fractures in relation to the other variables. This quality control study was performed in agreement with in-house ethical guidelines of the University Hospital of North Norway and the Helsinki Declaration.

\section{Results}

\section{Distribution of fractures}

A total of 593 patients with 691 fractures were registered. One patient had fractures in both hands, in $46 \%$ of the patients the right hand was involved and in $54 \%$ the left hand. There were 80 (12\%) carpal, 200 (29\%) metacarpal and 411 (59\%) phalangeal fractures (figure 1).

Sixty-four patients (11\%) had fractures in the carpal bones with 29 triquetral and 26 scaphoid fractures. In 183 patients (31\%) there were fractures in the metacarpal bones. A total of 99 fractures $(14 \%)$ were seen in the $5^{\text {th }}$ metacarpal 
bone, 46 of these (46\%) were sub-capital. In the left hand $64 \%$ of the metacarpal fractures were seen in the $5^{\text {th }}$ metacarpal bone, in the right hand this percentage was $60 \%$.

Fracture of the $5^{\text {th }}$ proximal phalanx was the most common phalangeal fracture with at total of 65 fractures (9\%). Epiphyseal fracture was the most common fracture type in the $5^{\text {th }}$ proximal phalanx, seen in 22 of the 65 patients (34\%). There were 44 fractures (6\%) registered in the $1^{\text {st }}$ distal phalanx. Summarizing metacarpal and phalangeal fractures, $215(31 \%)$ were localized in the $5^{\text {th }}$ row.

\section{Seasonal variation}

The total number of fractures was evenly distributed over the year but there was a slight decrease in July and a marked decrease December, the highest number of fractures was registered in February (figure 2). Triquetral fracture was the only fracture type with a clear seasonal variation (figure 3). Of the 29 fractures, 21 were registered in the winter period from November to March. Of the 26 scaphoid fractures, 13 occurred in March, April and May.

\section{Weekday variation}

The hand fractures were evenly distributed over the weekdays. However, metacarpal fractures occurred more commonly during weekends, 96 of the 186 fractures occurred on Friday, Saturday or Sunday. The most prominent 
difference was seen for $5^{\text {th }}$ metacarpal fractures, 57 of the 99 fractures occurring during Friday to Sunday (figure 4).

\section{Age and gender differences}

Average patient age was 34 years, the median age was 30 years (range 1-96). There was an increased number of fractures in the age group 10 to 24 years, representing $35 \%$ of the patients with a total of 223 fractures. Metacarpal fractures were most commonly seen in the age group 15 to 24 years representing $31 \%$ of the metacarpal fractures. Finger fractures were most common in the age group 15 to 24 years representing $19 \%$ of these fractures. Among the 593 patients, 415 (70 \%) were men and 178 (30\%) were women. Among male patients, the highest frequency of fractures was seen in the age group 10 to 60 years, while adult female patients had most fractures in the age group 50 to 70 years (figure 5). With regard to triquetral fractures, only two patients were under the age of 40 . The other 27 triquetral fractures were evenly distributed in the age group above 40 .

\section{Trauma mechanism}

In $9 \%$ of the referral texts no trauma mechanism was specified. In $27 \%$ of the female patients and $23 \%$ of the male falling was the trauma mechanism. With regard to triquetral fractures, falling was the most common trauma mechanism (27\%). Sports injuries represented a total of 133 patients (22\%) and $53 \%$ of these were specified as ball games. There was a reduced incidence of sport 
induced fractures during spring and summer. In 51 patients (9\%) aggressive behavior was specified as the trauma mechanism. Of these patients, $75 \%$ were in the age group 10 to 24 years; there were 49 male and 2 female patients in this group. For subcapital fractures in the $5^{\text {th }}$ metacarpal bone aggression was the trauma mechanism in $30 \%$ of the cases and $95 \%$ of these patients were men. Similar results are reported in a study from the Norwegian city Drammen [12] and in other studies $[3,6,13]$.

Male patients were also strongly overrepresented in traumas caused by axe and saw accidents, 30 of these 31 patients were male. In children sport injuries, falling and crush injuries were the most common mechanisms. In children below the age of 15 , sport injuries were specified as the trauma mechanism in 51 of the 124 patients, 31 (61\%) of these were specified as ball game injuries. Only 38 fractures (6\%) were caused by occupational accidents, 20 (53\%) of these were crush injuries. Of the occupational injuries $56 \%$ happened to patients between 25 and 49 years of age.

A total of 121 (21\%) of the fractures were operated, these were distributed as 9 carpal, 52 metacarpal and 69 finger fractures. Among the operated fractures 76 (60\%) were intra-articular metacarpal or finger fractures. Only $26(4.4 \%)$ of the patients were registered as treated with closed fracture reduction without surgery, evenly distributed between finger and metacarpal fractures. 


\section{Discussion}

Our results showed that finger fractures were more common than metacarpal bone fractures, while carpal fractures were the least common hand fractures. This is in accordance with other studies $[1,2,5,9]$. In our material, the $5^{\text {th }}$ finger in general and the $5^{\text {th }}$ metacarpal bone in particular was more prone to injury than the other fingers, a result also reported by others $[2-4,12]$.

Age and gender distribution in our material was in accordance with several other studies. In our study mean age was 34 years, while median age was 30 years. Earlier studies have shown a median age of 31-32 years and median age 27 years $[2,8]$. Similar to other studies, we also registered a high number of hand fractures in the age group 10 to 24 years peaking between 10 and 14 years. The age and gender distribution visualized in figure 5 has been reported in several studies $[1,3,4,14]$. Metacarpal fractures were most common in the age group 15-24 years representing $31 \%$ of the metacarpal fractures, quite similar to the results reported by Chung [9].

A study from the Netherlands [2] did not reveal any major seasonal variations in the incidence of hand fractures, except a small reduction during the summer months. A Canadian study [8] reported increased incidence during the spring and summer seasons when the climate favors outdoor activities. Interestingly, our study showed a distinct seasonal variation for triquetral fractures peaking during the winter months with falling as the dominating trauma mechanism. 
This prominent increase during the winter season may be explained by slippery winter conditions and polar darkness.

We found that $70 \%$ of all patients with hand fractures were males, other studies report $59-75 \%[2-5,7,8,14,15]$. In the age group 5 to 54 years, male patients had more hand fractures than females. Males were also overrepresented with regard to $5^{\text {th }}$ metacarpal fractures. These males obtained their fractures most frequently during weekends with aggression as the most common cause. This association is not restricted to the Arctic region, as Gudmundsen et al. reported similar results [12].

After the age of 55, women had almost as many hand fractures as men. Partially these differences may by explained by the fact that male patients more often have occupations involving the use of machines and mechanical work that involve a larger risk for hand injuries, and that this risk factor is eliminated after retirement. Postmenopausal women are also more prone to have osteoporosis than younger women. Elderly men may be prone to less aggression and risk taking behavior than younger men.

Others have studied factors as age, gender and different trauma mechanisms such as occupation and sports in relation to hand fractures. Our results show great similarity regarding these factors. All these studies, including our study, have been performed in Western industrialized countries with quite comparable culture and way of life. The only factor differentiating Northern Norway from the other geographical locations is the Arctic climate. We postulate that the main 
difference in distribution of hand fractures found in our study as compared to other studies is caused by the Arctic climate conditions.

One limitation of this study is selection bias as only patients that were referred to the university hospital have been included in the study. Although the university hospital is the only hospital in the region with a hand surgery unit, some patients with hand fractures are treated at local hospitals. Another limitation is that data was collected only from the year of 2010. The seasonal variation of triquetral fractures may have occurred only in this particular year.

\section{Conclusion}

Generally, hand fractures were not related to particular seasons or weekdays in this material. The distribution of hand fractures was related to age and gender and some trauma mechanisms were dominating. Unlike other studies, our study showed a seasonal variation of triquetral fractures peaking during the winter. Falling was the dominant trauma mechanism and the Arctic conditions may be responsible for this seasonal variation.

\section{Conflict of interest statement}

None of the authors has any financial interest, and none of the authors has any conflicts of interest. 


\section{REFERENCES}

[1] Hove LM. Fractures of the hand. Distribution and relative incidence. Scand J Plast Reconstr Surg Hand Surg 1993;27:317-9.

[2] van Onselen EB, Karim RB, Hage JJ, Ritt MJ. Prevalence and distribution of hand fractures. J Hand Surg Br 2003;28:491-5.

[3] Packer GJ, Shaheen MA. Patterns of hand fractures and dislocations in a district general hospital. J Hand Surg Br 1993;18:511-4.

[4] Anakwe RE, Aitken SA, Cowie JG, Middleton SD, Court-Brown CM. The epidemiology of fractures of the hand and the influence of social deprivation. J Hand Surg Eur Vol 2011;36:62-5.

[5] Worlock PH, Stower MJ. The incidence and pattern of hand fractures in children. J Hand Surg Br 1986;11:198-200.

[6] Greer SE, Williams JM. Boxer's fracture: An indicator of intentional and recurrent injury. Am J Emerg Med 1999:357-60.

[7] Mahabir RC, Kazemi AR, Cannon WG, Courtemanche DJ. Pediatric hand fractures: a review. Pediatr Emerg Care 2001;17:153-6.

[8] Feehan LM, Sheps SB. Incidence and demographics of hand fractures in British Columbia, Canada: a population-based study. J Hand Surg Am 2006;31:1068-74.

[9] Chung KC, Spilson SV. The frequency and epidemiology of hand and forearm fractures in the United States. J Hand Surg Am 2001;26:908-15.

[10] Absoud EM, Harrop SN. Hand injuries at work. J Hand Surg Br 1984;9:211-5. 
[11] Oleske DM, Hahn JJ. Work-related injuries of the hand: data from an occupational injury/illness surveillance system. J Community Health 1992;17:205-19.

[12] Gudmundsen TE, Borgen L. Fractures of the fifth metacarpal. Acta Radiol 2009;50:296-300.

[13] Yeo G, Evans M. Fifth metacarpal fractures-Do only "Boxers" get them? (An epidemiology study). Abstracts / Injury, Int J Care Injured 2009;40S (2009):S17. [14] Larsen CF, Mulder S, Johansen AM, Stam C. The epidemiology of hand injuries in The Netherlands and Denmark. Eur J Epidemiol 2004;19:323-7. [15] Fetter-Zarzeka A, Joseph MM. Hand and fingertip injuries in children. Pediatr Emerg Care 2002;18:341-5. 


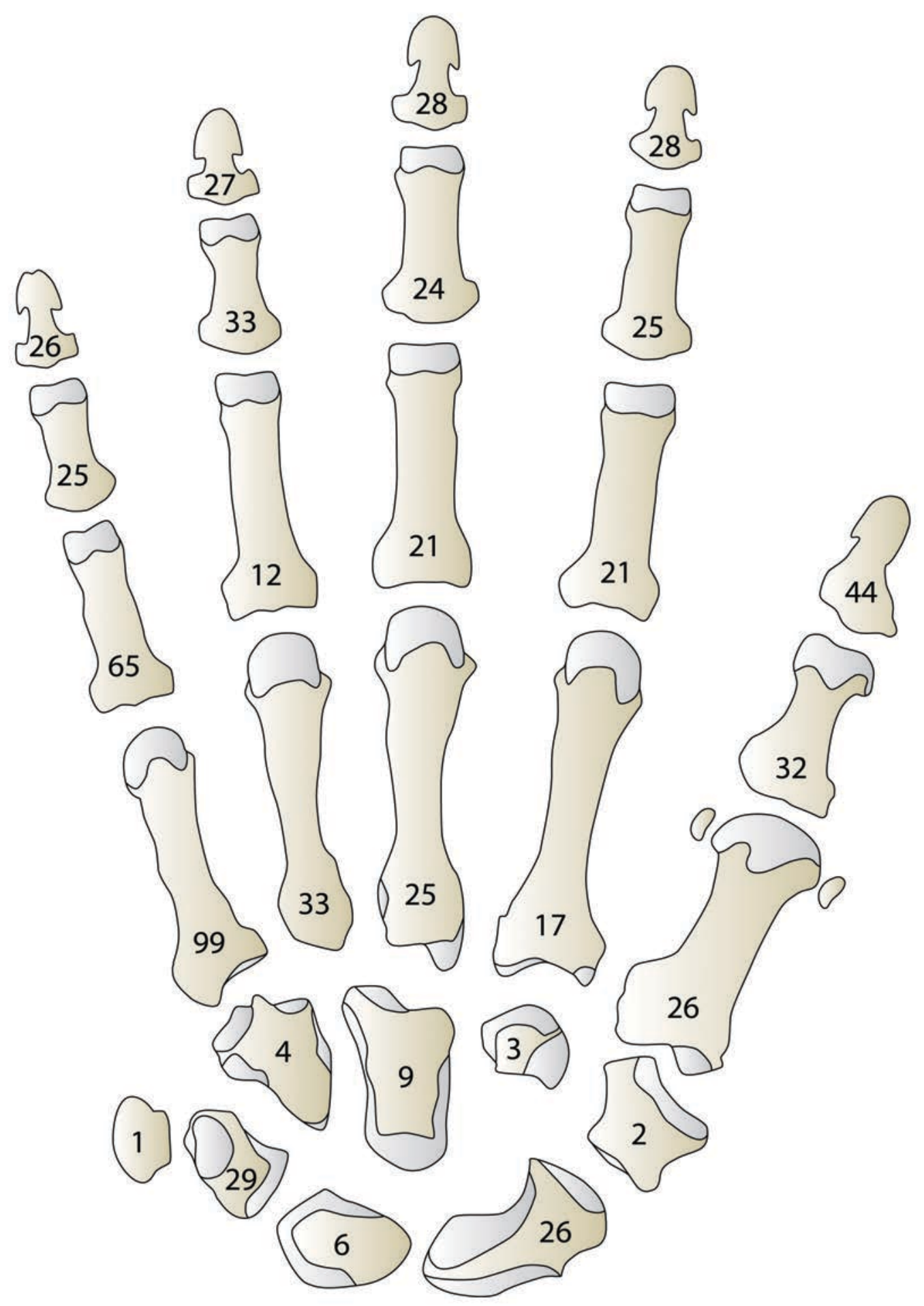

Figure 1

Distribution of fractures 
Hand fractures in Northern Norway

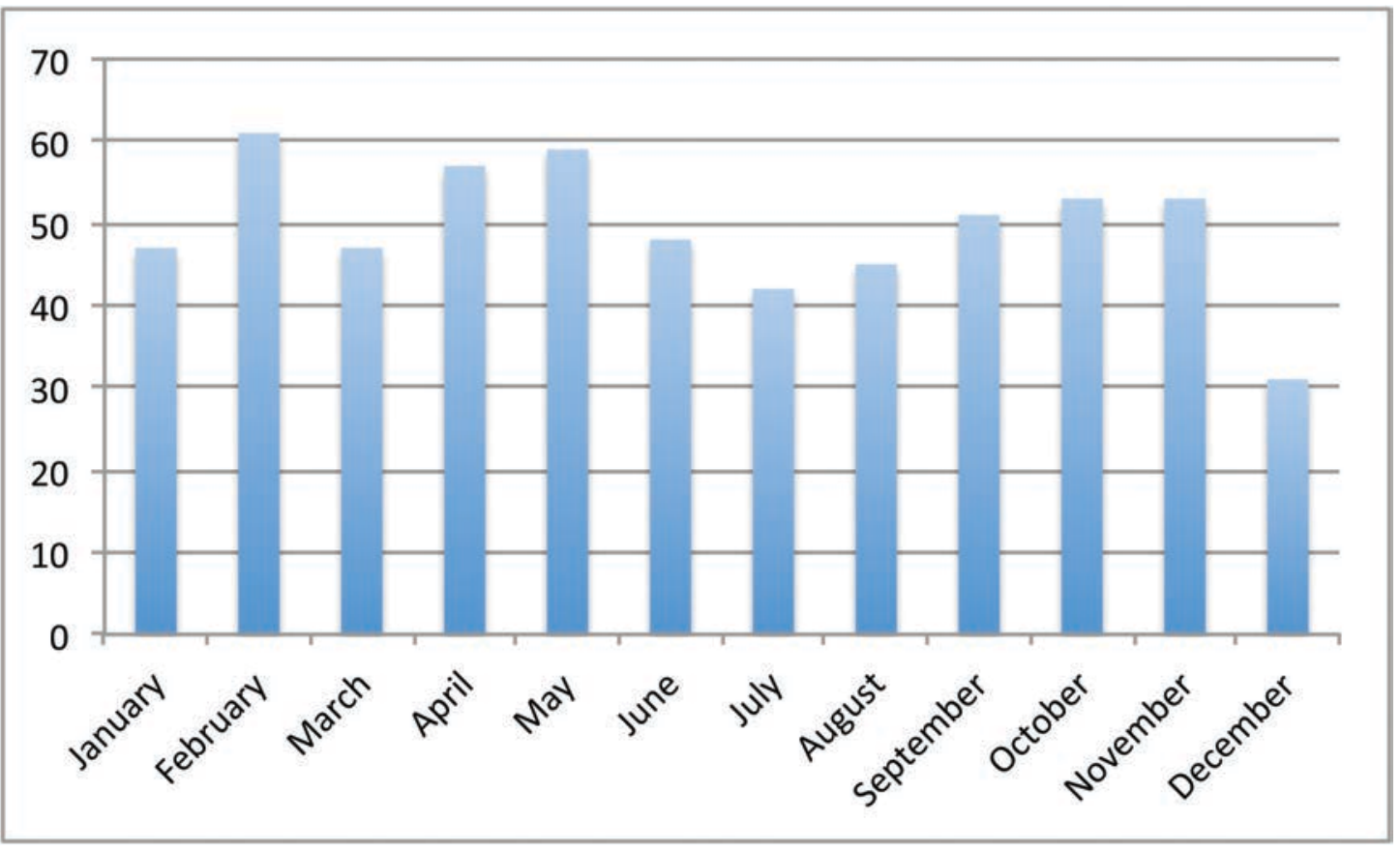

Figure 2

Seasonal variation of fractures 


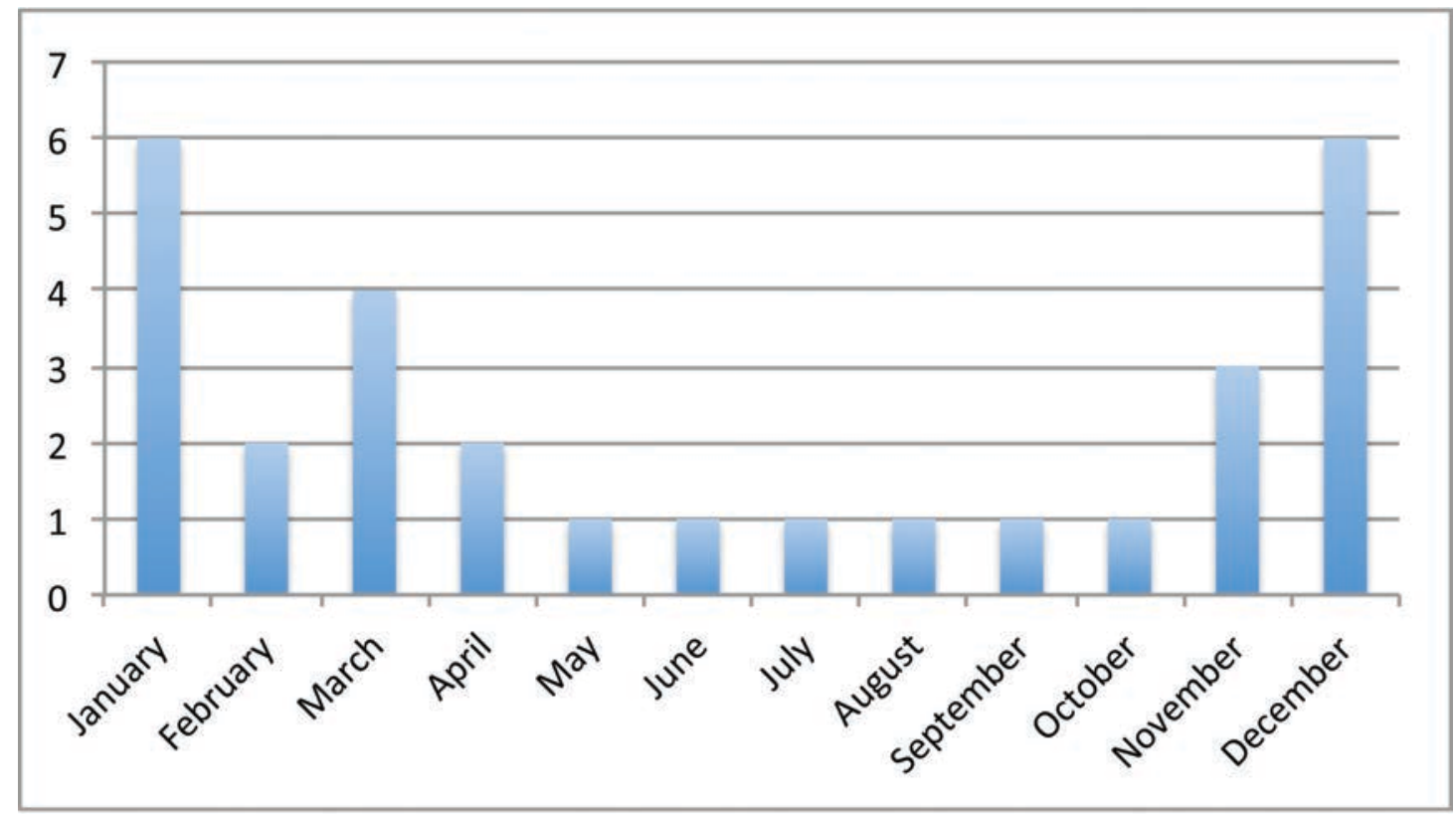

Figure 3

Seasonal variation of triquetral fractures 


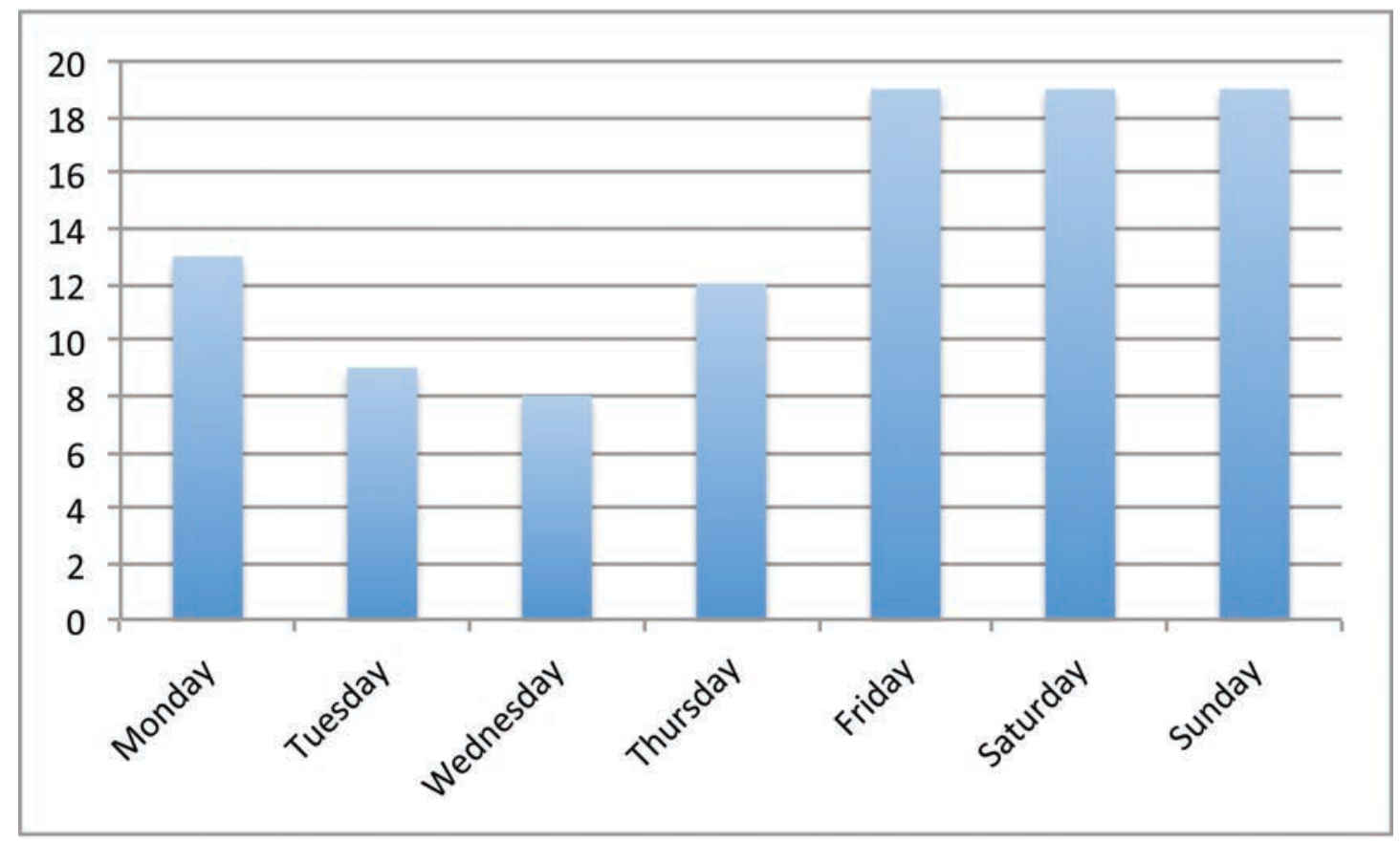

Figure 4

Weekday variation of 5 th metacarpal fractures 


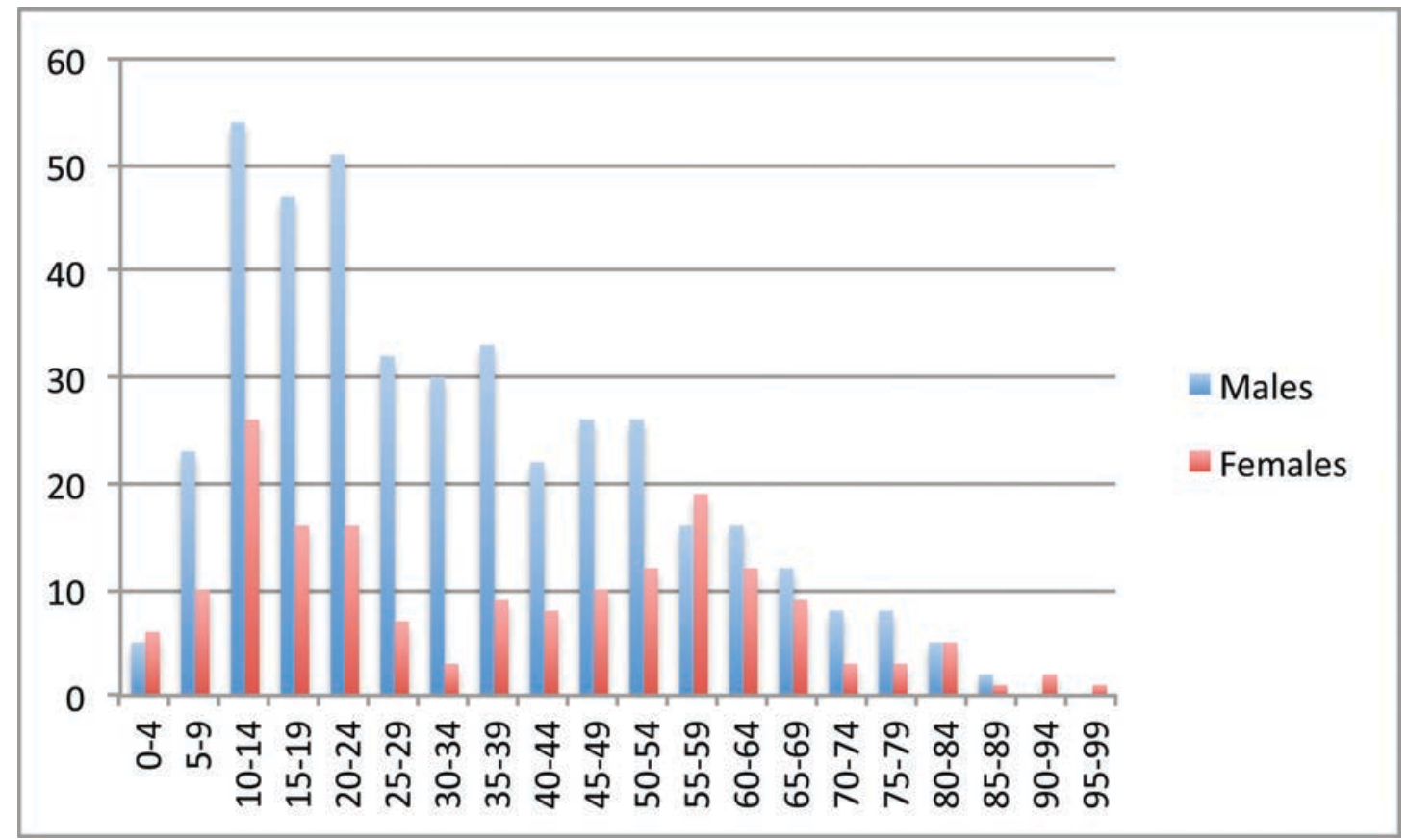

\section{Figure 5}

Age and gender distribution of finger fractures 